\title{
Matrix Singular Value Decomposition for Pole-Free Solutions of Homogeneous Matrix Equations as Applied to Numerical Modeling Methods
}

\author{
Vladimir A. Labay and Jens Bornemann, Senior Member, IEEE
}

\begin{abstract}
A general technique for solving homogeneous matrix equations as applied to numerical modeling procedures in microwave and millimeter-wave structures is introduced. By using singular value decomposition, well-known numerical problems related to poles and steep gradients in the determinant function are eliminated. The proposed technique is generally applicable, improves the accuracy and reliability of computed results, and significantly reduces the CPU time due to a more moderate behavior of the function to be analyzed. A dispersion characteristics example of a conductor-backed slotline MMIC structure illustrates the advantage of the pole-free formulation over conventional determinant calculations.
\end{abstract}

\section{INTRODUCTION}

$\mathbf{M}$ ANY applications of existing numerical techniques for the analysis of microwave and millimeter-wave structures lead to a homogeneous matrix equation in the form of

$$
(\boldsymbol{A}) \cdot \boldsymbol{x}=0,
$$

where $(\boldsymbol{A})$ is a complex matrix of size $m \times n(m \geq n)$ and $x$ is an $n$-element column vector, e.g., [1], [2]. In order to determine the solutions of this equation, it is common practise to vary a (complex or real) parameter $\gamma$ until

$$
\operatorname{det}(\boldsymbol{A})=0 \text { for } \gamma=\gamma_{0}
$$

This parameter $\gamma$ can be the propagation constant, the effective permittivity, or the cutoff frequency. The corresponding elements of the solution vector $x$ are required for the calculation of the characteristic impedance, the field distribution, or for three-dimensional analyses.

The accuracy with which (1) can be solved is directly related to the accuracy with which the zeros of $\operatorname{det}(\boldsymbol{A})$ can be detected. For most of the numerical techniques used [1],[2], however, this is a difficult task because of two factors. First, $\operatorname{det}(\boldsymbol{A})$ is a rapidly changing function with $\gamma$, containing not only poles and zeros in close neighborhood but also extremely steep gradients and, secondly, neither the pole nor the zero locations can be expressed analytically. Therefore, (1) has to be solved on a computer where any search algorithm to detect the zeros has to operate at small step widths, has to cope with inaccuracies introduced by the numerical limits of the

Manuscript received July 11, 1991; revised September 30, 1991.

The authors are with the Department of Electrical and Computer Engineering, P.O. Box 3055, University of Victoria, Victoria, BC, Canada V8W 3P6.

IEEE $\log$ Number 9105532. computer and, therefore, slows down the numerical solution of (1) significantly. Although these difficulties are well known, only few attempts to address and alleviate this problem for finline circuits have been reported so far.

In [3], a formulation based on pole-zero pairs is used. In [4], the poles are used to construct a pole-free function which then can be searched for its zeros. In both cases, the accurate determination of the pole locations is required in a first step in order to reliably detect the zeros in the second step. A wellknown possibility of reducing or even avoiding poles is to maintain the original equations as derived from the boundary conditions and refrain from any manipulations. Hence, a pole only occurs if one matrix elements goes to infinity. However, the resulting large matrix, which has to be solved for a given number of consecutive determinant zeros, often leads to unacceptably high CPU time requirements. A method that combines a small matrix size with a pole-free determinant function [5] exhibits an almost rectangular function shape, thus making great demands on the accuracy of the computer.

Therefore, this letter presents a general technique for solving the (complex) homogeneous matrix equation. The method is based on singular value composition which is a powerful algorithm for dealing with matrices that are either singular or else numerically very close to singular [6]. It is demonstrated that by detecting the minima of the minimum singular value, instead of the zeros of the system determinant, the presence of poles can be eliminated, thus significantly simplifying the search algorithm and increasing the accuracy of the computations. Moreover, the detected value of the minimum itself provides a clear indication for the accuracy achieved.

\section{THEORY}

The singular value decomposition of the matrix $(A)$ in (1) requires that

$$
(\boldsymbol{A})=(\boldsymbol{W})(\boldsymbol{S})(\boldsymbol{V})^{+},
$$

where $(S)$ is a diagonal matrix formed by the singular values in decreasing order, the columns of $(\boldsymbol{W})$ and $(\boldsymbol{V})$ are the left and right singular vectors of $(\boldsymbol{A})$, respectively, and + indicates the transposed conjugate [7]. (It should be mentioned that this procedure differs from the standard eigenvalue equation (A) $\cdot \boldsymbol{x}=\lambda \cdot \boldsymbol{x}$ which requires the solution of $\operatorname{det}\{(\boldsymbol{A})-\lambda$. $(I)\}=0$. In this letter, we are not concerned with eigenvalues and eigenvectors. We rather solve for the singular values of a 
necessarily singular matrix $(\boldsymbol{A})$ and the corresponding solution vector $\boldsymbol{x}$ (cf. (1)). For a more detailed description, which includes Fortran source codes, the reader is referred to [6]).

$\operatorname{det}(\boldsymbol{A})$ is still a function of $\gamma$, but instead of detecting zeros of $\operatorname{det}(\boldsymbol{A})$, the algorithm now searches for the minima of the last element $\sigma_{m}$ of the diagonal matrix $(\boldsymbol{S})$, which for $\gamma=\gamma_{o}$ yields

$$
\sigma_{m}=\overrightarrow{0} \text {. }
$$

It should be noted that (2) and (4) are equivalent conditions, that is

$$
\sigma_{m}=0, \text { if and only if } \operatorname{det}(\boldsymbol{A})=0 .
$$

As will be demonstrated in the next section, detecting the minima of $\sigma_{m}$ is extremely simple and far more reliable than the search for zeros of $\operatorname{det}(\boldsymbol{A})$. Moreover, if (5) is satisfied, the last column of $(\boldsymbol{V})$ automatically contains the corresponding solution vector $\boldsymbol{x}$ (cf. (1)). For a square matrix $(\boldsymbol{A})$, which is the case for most numerical problems related to microwave transmission lines, the singular values $\sigma_{1}$ to $\sigma_{m}$ are real. In more general applications $(m>n)$, the singular values can be made real by simultaneous diagonalization, e.g., [8].

Although the search for a minimum is somewhat more difficult than for a zero, the advantage of singular value decomposition lies primarily in the fact that the procedure provides numerical stability independent of the presence of poles or steep gradients since it is especially designed for singular or near-singular matrices [6], [7]. Moreover, it automatically provides the user with the corresponding solution vector whose calculation, using the conventional search for $\operatorname{det}(\boldsymbol{A})$, might be slightly inaccurate depending on the accuracy of the detected zero. Again, for the singular value decomposition itself, we would like to refer the reader to [6] since its mathematical formulation is beyond the scope of this letter.

\section{RESULTS}

The advantage of the singular value decomposition in (3), (4) over the more commonly used zero search of $\operatorname{det}(\boldsymbol{A})$ is demonstrated for the case of a complex homogeneous equation derived from a scattering-type transverse resonance formulation [9] as applied to a conductor-backed MMIC slotline structure [10]. Fig. 1(a) compares the system determinant (solid line) with the minimum singular value (dashed line) versus the normalized propagation constant. The zeros of the determinant, which coincide with the minima of the minimum singular value, correspond to the normalized propagation constants of the fundamental (Fig. 1(a), right) and the next seven higher order modes (from right to left). Due to the presence of poles and steep gradients in the determinant function, the accurate and reliable detection of the determinant zeros on a computer with limited accuracy is more difficult than the search for the minima of $\sigma_{m}$.

Fig. 1(b) shows an enlargement of Fig. 1(a) that focuses on one of the critical regions around $k_{z} / k_{o}=3.14$. Numerical investigations have shown that if the circuit parameters (cf. legend of Fig. 2) or the frequency are changed, the two zeros between $k_{z} / k_{o}=3.12$ and 3.13 move toward each

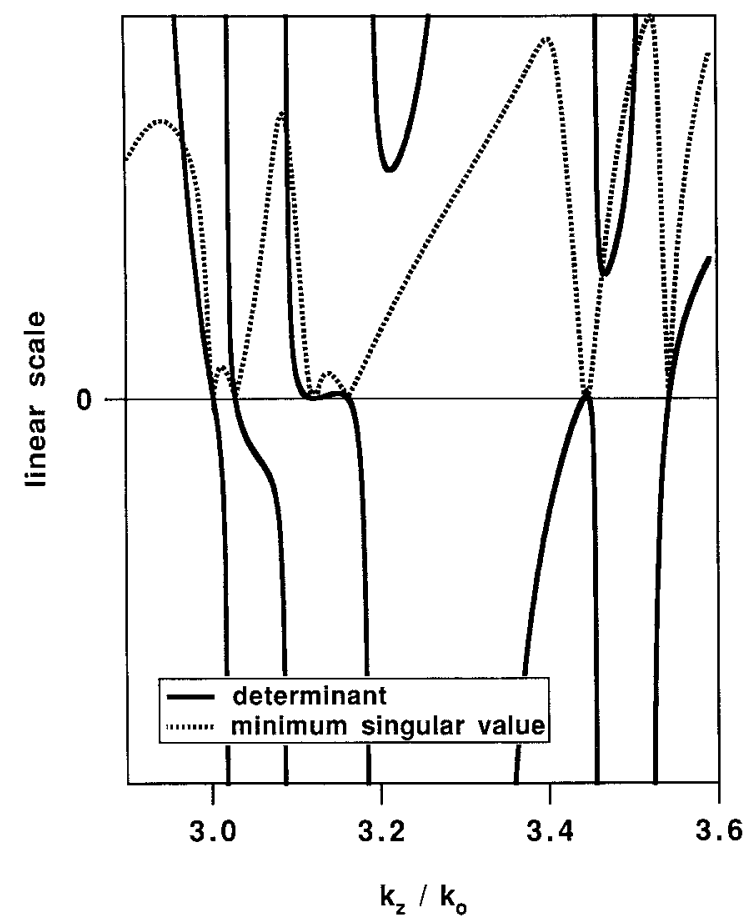

(a)

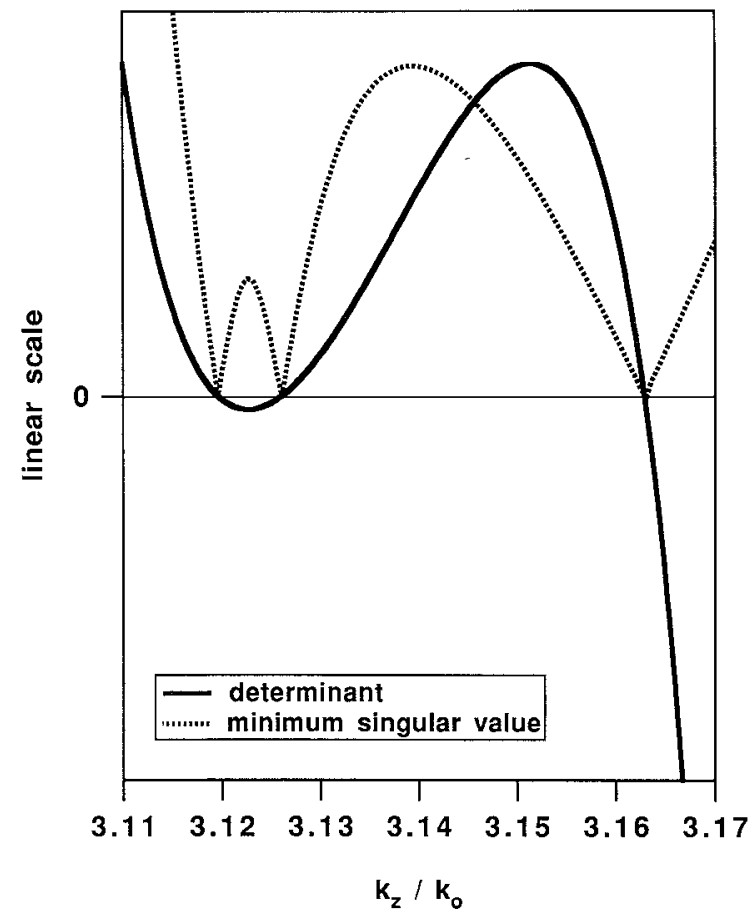

(b)

Fig. 1. (a) Behavior of the system determinant (solid line) and minimum singular value (dashed line) for a modified transverse resonance formulation of an MMIC conductor-backed slotline structure. Zeros correspond to the normalized propagation constants of fundamental and higher order modes. (b) Enlargement of (a) in the region around $k_{z} / k_{\mathrm{o}}=3.14$.

other until a zero can no longer be detected. This is a potential disadvantage when using the determinant function. The singular value decomposition, however, clearly identifies this point as two solutions of the homogeneous equation system (1). In cases like this, not only the minimum but also 
the next singular value vanishes. In the practical application of, e.g., a dispersion diagram, this means that the method permits the detection of two modes having identical propagation constants at a certain frequency whereas, in comparison, these modes would not be detected using the conventional search for the zeros of the determinant.

Finally, Fig. 2 shows a complete dispersion diagram of the slotline structure including reference values presented in [10]. Although the CPU time for the calculation of one single singular value decomposition is slightly higher than that for the corresponding determinant, the overall computation for the complete diagram of Fig. 2 could be reduced by a factor of 3.5 compared to the determinant search algorithm used in [9]. This is due to the absence of poles and the general shape of the singular value function, which offers the possibility of using variable step widths during the search for the minima. An additional advantage of this method is that an increase of the minima of $\tau_{\mathrm{m}}$ indicates a reduced accuracy in the computation. This can be incorporated into a software package in order to inform the user of possible errors due to complex matrix operation or limited precision of the computer. So far, detected minima more than 15 orders of magnitude below the maxima have provided reliable results.

\section{CONCLUSION}

Singular value decomposition eliminates numerical problems in determining solutions of homogeneous matrix equations as related to numerical modeling procedures for microwave and millimeter-wave structures. Instead of poles and steep gradients observed in the commonly used determinant function, the general technique proposed here produces a simple and easy-to-analyze function whose minima correspond to the solutions sought. Its general applicability, its reduction in CPU time, its improvement in accuracy, its reliability on the computed results and its ability to locate zeros of higher order make this technique an attractive and powerful tool for electromagnetic field modeling.

\section{ACKNOWLEDGMENT}

The authors would like to thank Profs. R. Vahldieck and W.-S. Lu for helpful suggestions and discussions.

\section{REFERENCES}

[1] R. Sorrentino, Ed., Numerical Methods for Passive Microwave and Millimeter-Wave Structures. New York: IEEE Press, 1989.

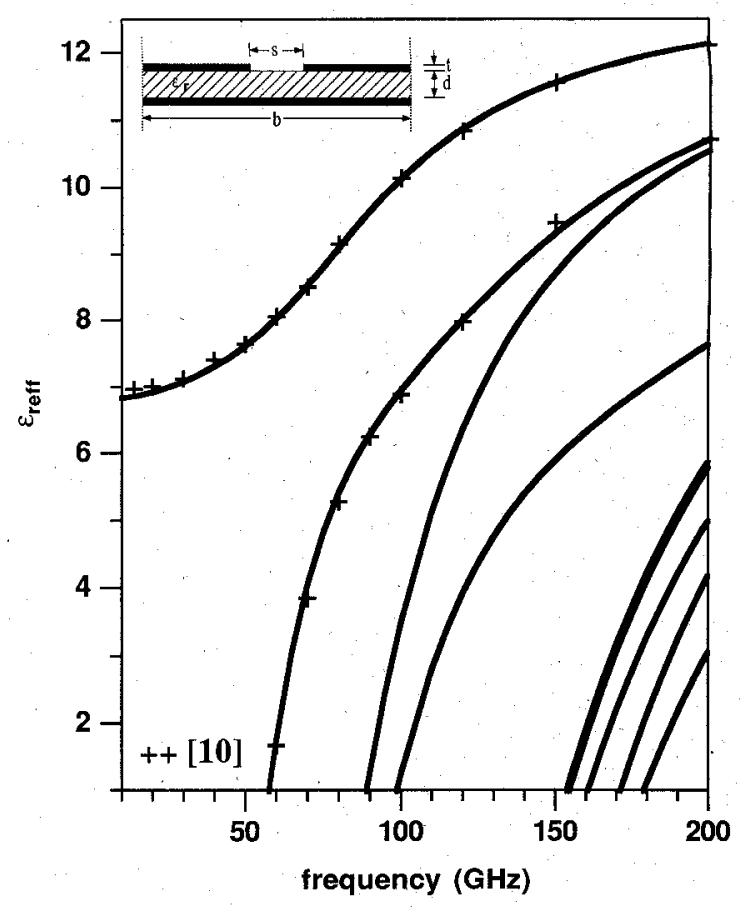

Fig. 2. Dispersion diagram $\left(\varepsilon_{\text {reff }}=\left(k_{z} / k_{o}\right)^{2}\right)$ of conductor-backed slotline (++ [10]). Dimensions: $b=840 \mu \mathrm{m}, s=40 \mu \mathrm{m}, t=3 \mu \mathrm{m}$, $d=600 \mu \mathrm{m}, \varepsilon_{r}=12.9$.

[2] T. Itoh, Ed., Numerical Techniques for Microwave and Millimeter-Wave Passive Structures. New York: John Wiley, 1989.

[3] C.A. Olley and T.E. Rozzi, "Systematic characterization of the spectrum of unilateral finline," IEEE Trans. Microwave Theory Tech., vol. MTT34, pp. 1147-1156, Nov. 1986.

[4] A.S. Omar and K. Schünemann, "Pole-free formulation for the determination of finline modes," SBMO Int. Microwave Symp. Proc., Rio de Janeiro, Brazil, July 1987, pp. 477-481.

[5] J. Bornemann, "Comparison between different formulations of the transverse resonance field-matching technique for the three-dimensional analysis of metal-finned waveguide resonators," Int. J. Numer. Modeling, vol. 4, pp. 63-73, Mar. 1991.

[6] W.H. Press, B.P. Flannery, S.A. Teukolsky, and W.T. Vetterling, $\mathrm{Nu}$ merical Recipes - The Art of Scientific Computing (Fortran Version). New York: Cambridge Univ. Press, 1989.

[7] R.A. Horn and C.A. Johnson, Matrix Analysis. New York: Cambridge Univ. Press, 1985.

[8] P.M. Gibson, "Simultaneous diagonalization of rectangular complex matrices," Linear Algebra and Its Applications, vol. 9, pp. 45-53, 1974.

[9] J. Bornemann, "A scattering-type transverse resonance formulation and its application to open, conductor-backed and shielded (M)MIC slotline structures," in IEEE MTT-S Int. Microwave Symp. Dig., June 1991, pp. 695-698.

[10] W. Heinrich, "The slotline in uniplanar MMIC's: Propagation characteristiçs and loss analysis," in IEEE MTT-S Int. Microwave Symp. Dig., May 1990, pp. 167-170. 